\title{
Demonstration of Cavity-Enhanced Optical Parametric Chirped-Pulse Amplification System at High Repetition Rate
}

\author{
Aleem Siddiqui ${ }^{1, *}$, Kyung-Han Hong, ${ }^{1}$ Jeffrey Moses, ${ }^{1}$ Jeff Chen, ${ }^{1}$ F. Ömer Ilday ${ }^{2}$, and Franz X. Kärtner ${ }^{1}$ \\ ${ }^{1}$ Department of Electrical Engineering and Computer Science and Research Laboratory of Electronics, \\ Massachusetts Institute of Technology (MIT), Cambridge, MA 02139, USA \\ ${ }^{2}$ Department of Physics, Bilkent University, Cankaya, Ankara 06800, Turkey \\ *Email: siddiq@mit.edu
}

\begin{abstract}
First experimental demonstration of cavity-enhanced OPCPA at $78 \mathrm{MHz}$ with $<1 \mathrm{~W}$ of pump power is presented. For comparison, we demonstrated saturated gain in a single-pass experiment from 6-W Yb-fiber pump and Er-fiber signal sources.
\end{abstract}

\section{Introduction}

Recently, optical parametric amplification (OPA) and optical parametric chirped-pulse amplification (OPCPA) have emerged as a means of providing intense few-cycle optical pulses for applications including ultrafast timeresolved spectroscopy, and high-harmonic generation. Ultrabroadband OPA and OPCPA sources, mostly pumped by Ti:sapphire- or Nd-based regenerative and/or multipass amplifiers, have been limited to repetition rates in the100 $\mathrm{kHz}$ range [1]. Only recently, OPA systems with repetition rates in the $\mathrm{MHz}$ range have emerged, exploiting new ultrashort pump sources based on double clad Yb-fiber, cryogenically cooled Yb-doped or thindisk Yb-doped amplifiers with the design emphasis on the scaling of the average pump power to maintain high peak intensities for high signal gain [2-4]. Further scaling of the repetition rate and average power in parametric amplifiers - while maintaining peak intensity - is highly desirable for many applications.

Cavity-enhanced OPCPA (C-OPCPA) [5] has been proposed for an efficient OPCPA process with low-energy, high-average-power pump sources at the repetition rate of $<1 \mathrm{MHz}$. More recently, we explored the C-OPCPA concept in a high-repetition-rate $(\sim 100 \mathrm{MHz})$ scheme utilizing the full repetition rate of the seed lasers [6]. In the latter C-OPCPA, pump pulses are coherently combined in a low finesse enhancement cavity transparent to signal and idler, containing an OPA crystal in which a synchronized signal beam is amplified via OPCPA. The cavity passively shapes the intra-cavity pump profile to attain more optimal conversion, overcoming limitations set by the bell-shaped pump intensity profile and the time-varying wave-vector mismatch of the interacting pulses. We have numerically shown that the passive pulse shaping leads to both gain bandwidth enhancement and increased conversion. In this work we present a first demonstration of a fiber-based C-OPCPA system. With less than $1 \mathrm{~W}$ of pump power and at 78MHz, accounting for coupling loss, we demonstrate conversion efficiency approaching $50 \%$. This result opens the possibility for OPCPA pumped directly with an unamplifed pulsed laser source.

\section{Experimental setup and results}

Figure 1 illustrates the optical layout of the C-OPCPA system developed thus far. A similariton Yb-doped fiber laser (YDFL) and a soliton Er-doped fiber laser (EDFL) are used as an OPCPA pump source at $1.03 \mu \mathrm{m}$ and an OPCPA seed at $1.56 \mu \mathrm{m}$, respectively, both with 78-MHz repetition rate. The YDFL seed is amplified in a conventional femtosecond chirped-pulse amplification system consisting of a grating stretcher, a home-built Ybdoped fiber pre-amplifier, a commercial 8-W fiber amplifier, and a dielectric grating compressor. This system provides 5-nm bandwidth (BW) pump pulses with durations down to 500 fs with up to 6-W average power for the OPCPA pumping. The EDFL output pulses are stretched to match the duration of the pump pulses using $5 \mathrm{~m}$ of fiber for the OPCPA seed. The pump and stretched seed pulses are simultaneously injected into a 78-MHz cavity resonant with the pump pulses and containing a 20mm PPLN crystal anti-reflection (AR) coated at the pump wavelength for parametric amplification. Finally, the amplified seed pulses can be compressed with a silicon prism pair. The pump and signal sources are electronically locked via feedback to a piezo-mounted cavity mirror in the EDFL. Less than 50 fs of timing jitter was observed between the two lasers. The YDFL cavity is separately locked to the enhancement cavity via a Hänsch-Couillaud (HC) scheme.

In C-OPCPA, an enhancement cavity is used to recycle unused pump light due to an inefficient intracavity conversion process. Due to the large signal chirp, each temporal coordinate arrives at steady state independently, and temporal regions with low conversion (due to locally low pump intensity and/or large wave-vector mismatch) exhibit lower loss with a pump light and thus develop a higher pump intensity that compensates for the initially low gain. The simulation results in Fig. 2 [6] reveal the underlying mechanism that leads to both gain and BW improvements. A winged intracavity pump profile develops to compensate the increased wavevector mismatch and reduced seed and pump intensities away from $t=0$ where the wave-vector mismatch is zero. Each temporal coordinate settles to a value of intracavity power, reflected power, and nonlinear loss that simultaneously satisfies the conditions for the buildup power of a passive cavity and parametric conversion where optical feedback occurs due to the coupling of intracavity power and nonlinear loss. At two temporal coordinates, the cavity loss equals the input coupling and impedance matching exactly. Overall good impedance 


\section{TuC1.pdf}

matching is achieved over the whole pulse duration resulting in increased conversion and bandwidth. A conversion of $68 \%$ with 103-nm BW was achieved, exceeding the single-pass phase matching BW of $24 \mathrm{~nm}$ by a factor of four while an optimal single-pass OPCPA with similar parameters yields only $43 \%$ conversion with 52$\mathrm{nm}$ BW due to saturation needing 25-W pump power.

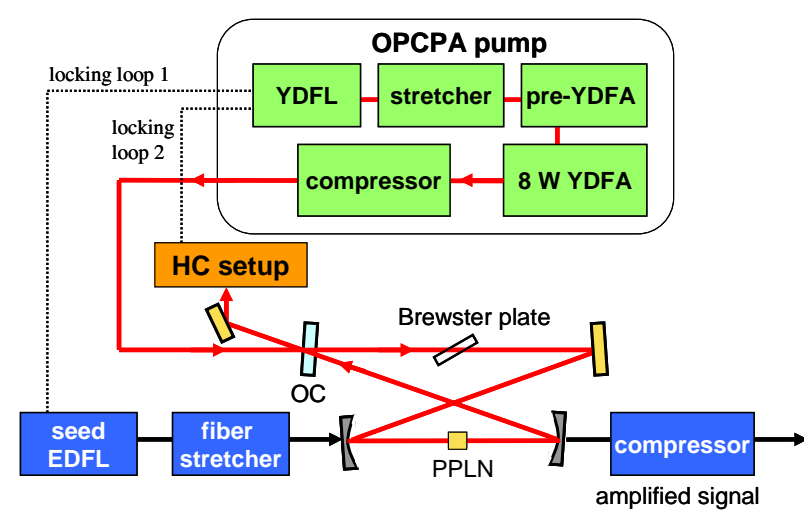

Fig. 1 Schematic of C-OPCPA device for proof-of-principle experiment: OC, output/input coupler; YDFL/A, Yb-doped fiber laser/amplifier; EDFL, Er-doped fiber laser

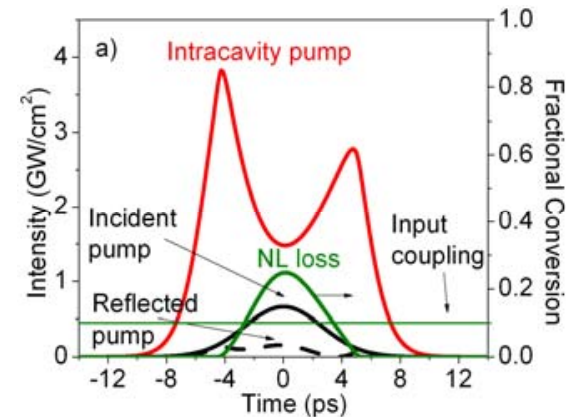

Fig. 2. C-OPCPA simulation for narrowband 5-W, 6-ps Gaussian pump at $1.03 \mu \mathrm{m}, 1 \mathrm{~mW}, 6$-ps signal pulses at $1.55 \mu \mathrm{m}$ with $100-\mathrm{nm} \mathrm{BW}$, a cavity input coupling, $\mathrm{T}=$ $10 \%$, and with a 5-mm-long PPLN crystal (Red) Intracavity pump profile. (Black) Incident pump profile. (Black, dashed) Reflected pump power profile. (Olive) Nonlinear loss (NL) at each temporal coordiante.

We performed single-pass parametric amplification using the same C-OPCPA setup of Fig. 1 without closing the enhancement cavity. Fig. 3 shows the amplified signal spectrum, the super-florescence, and the seed spectrum of the single-pass OPCPA. The pump and signal sources are focused into a 20-mm-long PPLN crystal with $120 \mu \mathrm{m}$ of Gaussian beam diameter. This spot size is close to that of the mode in the closed cavity. With 6 $\mathrm{W}$ of $500 \mathrm{fs}$ pump pulses, we were able to convert $8 \%$ of the pump power into $35 \mathrm{~nm}$ of BW of the chirped signal at $78 \mathrm{MHz}$. Accounting for the idler at $3.03 \mu \mathrm{m}$ which was fully absorbed in a BK7 collimating lens, we estimate that the total pump depletion was $12 \%$. Thus, with the same $6 \mathrm{~W}$ of intracavity power, the nonlinear loss is expected to be $12 \%$, as well. The measured linear loss from the additional components in the cavity, mirrors and AR coatings on the PPLN is 2\%. By reducing the intracavity pump power to allow $8 \%$ conversion, the total loss can be tuned to $10 \%$, matching the value of the output coupler such that on average the impedance matched condition is met. Importantly, this experiment shows that sufficient amplification can be achieved before crystal damage occurs at the desired power levels.

When the full C-OPCPA apparatus is employed with only $820 \mathrm{~mW}$ of incident pump, $60 \mathrm{~mW}$ are depleted into the signal and we estimate $30 \mathrm{~mW}$ are depleted into the idler. The resulting signal spectrum is shown in Fig 3. Under present alignment conditions only $1 / 4$ of the incident pump energy is

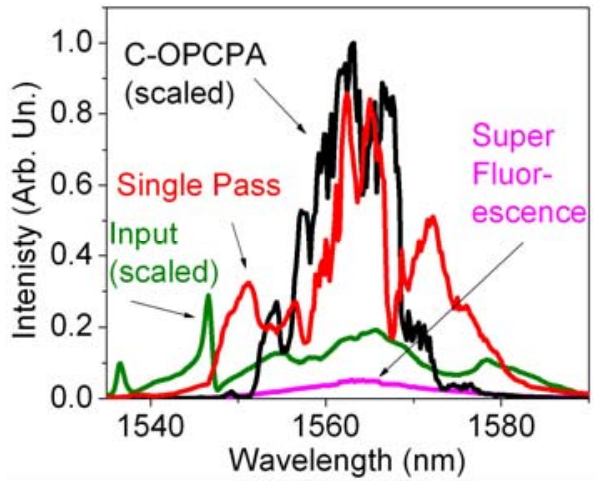

Fig. 3 Single-pass OPCPA result: (Red) amplifed signal, (Black) C-OPCPA amplifed signal (Blue) seed spectrum from EDFL. (Magenta) Superflorescence noise spectrum coupled into the cavity mode. Only the coupled light is able to contribute to the nonlinear conversion. Thus with an effective available pump energy of only $205 \mathrm{~mW}$ we are able to achieve nearly $44 \%$ pump depletion for a $78 \mathrm{MHz}$ source. We note that, allowing an enhancement of 10 , the intracavity conversion is only $4.4 \%$, however, good average impedance matching enables the dramatic conversion efficiency improvement with respect to the coupled incident pump. Further improvements in overall conversion efficiency can be achieved in our system with improvements in transverse spatial mode coupling. Additionally we expect to demonstrate BW enhancement by increasing the pump pulse duration to match the experimentally reachable parameters in the above simulation. Further experimental progress will be reported.

\section{References}

[1] G. Cerullo and S. De Silvestri, Rev. Sci. Instrum. 74, 1 (2003).

[2] T. Eidam, S. Hanf, E. Seise, T. V. Andersen, T. Gabler, C. Wirth, T. Schreiber, J. Limpert, and A. Tünnermann, Opt. Lett. 35, 94 (2010). [3] E. Innerhofer, T. Südmeyer, F. Brunner, R. Häring, A. Aschwanden, R. Paschotta, C. Hönninger, M. Kumkar, and U. Keller, Opt. Lett. 28, 367-369 (2003).

[4] K.-H. Hong, A. Siddiqui, J. Moses, J. Gopinath, J. Hybl, F. Ö. Ilday, T. Y. Fan, and F. X. Kärtner, Opt. Lett. 33, 2473 (2008).

[5] F. O. Ilday and F. X. Kärtner, Opt. Lett. 31, 637 (2006).

[6] A. M. Siddiqui, J. Moses, K.-H. Hong, and F. X. Kärtner, CLEO/QELS 2009 (Baltimore, Maryland, May 31- June 5, 2009) CWL1. 